\title{
Formation and Change of Planetary Magnetic Field
}

\author{
Cuixiang Zhong \\ Department of Physics, Jiangxi Normal University, Nanchang, China
}

\section{Email address:}

cuixiang_zhong@163.com

\section{To cite this article:}

Cuixiang Zhong. Formation and Change of Planetary Magnetic Field. American Journal of Astronomy and Astrophysics. Vol. 8, No. 3, 2020, pp. 61-65. doi: 10.11648/j.ajaa.20200803.13

Received: August 26, 2020; Accepted: September 14, 2020; Published: September 25, 2020

\begin{abstract}
The influential theories about the origin of planetary magnetic field hold that the planetary magnetic field is produced by the flow of conductive fluid in the core. But these hypotheses have serious defects, unable to explain the inhomogeneity of the spatial distribution of planetary magnetic field and its changing characteristics with time. Thus, the author analyzed the formation and evolution of solar system planets as well as their internal structures and external environments, and has found the formation mechanism and change law of various planetary magnetic fields. The polar vortices at Earth's North and South Poles can produce spiral currents, which then form a magnetic dipole at Earth's North and South Poles respectively. Mercury is about $70 \%$ metal and 30\% silicate, so it has been magnetized by the Sun's magnetic field. Venus' rotation speed is too slow to form polar vortices needed to produce dipole magnetic field, and Venus is far away from the Sun, causing the solar magnetic field has little effect on the magnetization of Venus, so Venus' magnetic field is extremely weak. During the first 500 million to1 billion years of Mars formation, polar vortices existed for a long time. The dipole magnetic field produced by the polar vortices has a long-term magnetization effect on the Mars' surface, therefore a magnetized crust on the surface of Mars has been formed. But with the heat inside the Mars accumulated to a certain extent, a large part of Mars Polar ice sheet melt into water. The melting of the Martian polar ice sheet greatly weakened the polar vortex and therefore the magnetic field. Especially, in the northern part of Mars, there are large-scale lava activities in the lowlands or volcanic areas, the ice sheet melted a lot there, therefore no polar vortex could be formed, causing the dipolar field disappeared. During Jupiter's rapid rotation, a series of strong polar vortices are produced at the poles of Jupiter. These vortices contain a series of strong spiral currents, which can form a series of strong dipole magnetic fields. The superposition of these dipole magnetic fields form the original magnetic field of Jupiter. But some of Jupiter's massive moons can induce some sub cyclones from the Jupiter's vortices, these sub cyclones form powerful cyclones by absorbing dense clouds and generate some new magnetic fields, which are superimposed on the original magnetic field to form more complex magnetic field of Jupiter.
\end{abstract}

Keywords: Mercury, Venus, Earth, Mars, Jupiter, Planets, Magnetic Fields

\section{Introduction}

Planetary magnetic field refers to the magnetic field around the planet, but the influential theories about the origin of planetary magnetic field follow the internal dynamo theory of geomagnetic field, that is, they hold that the planetary magnetic field is produced by the flow of conductive fluid in the core $[1,2]$. But these hypotheses have serious defects, unable to explain the inhomogeneity of the spatial distribution of planetary magnetic field and its changing characteristics with time. Thus, the author analyzed the formation and evolution of solar system planets as well as their internal structures and external environments again, and has found the formation mechanism and change law of various planetary magnetic fields.

\section{Formation and Change of Geomagnetic Field}

Many hypotheses have been put forward on the origin of the geomagnetic field. Among them, dynamo theory of the geomagnetic field is more influential than others, but it is still unable to explain the inhomogeneity of the spatial distribution of planetary magnetic field and its changing characteristics with time. Hence, it has serious defects, failed to become a 
truly scientific theory. Recently, the author analyzed the formation and evolution of the Earth as well as its internal structure and external environment again, and has found the origin of the geomagnetic field.

The Earth is surrounded by a thick atmosphere, but the strong centrifugal force produced by the rapid rotation of the Earth makes the clouds over the equator and low latitudes tend to move away from their orbits to the South pole or the North Pole. Since the radius of the two poles of the Earth is less than the radius of the equator and other places, and the gravitational force is inversely proportional to the square of the distance, the gravitational attraction of the polar position is greater than that of other locations. When the clouds move above the polar regions, they are easily attracted by the gravitational pull of the polar regions, after inhaling cold air, they condense into thick clouds and sink gradually. Many polar-plunging clouds form a strong circulation around the pole as the Earth rotates, that is polar vortex, as is shown in Figure 1. The Earth has two group of vortices, located at the South pole and the North pole respectively, which can span troposphere and stratosphere. When the Arctic is in summer and its vortex structure become weaker than in winter, the Antarctic is in winter and its vortex become stronger than in summer, and vice versa.

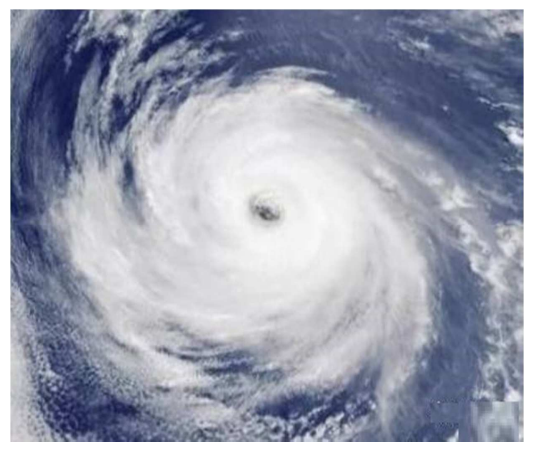

Figure 1. Earth's polar vortex.

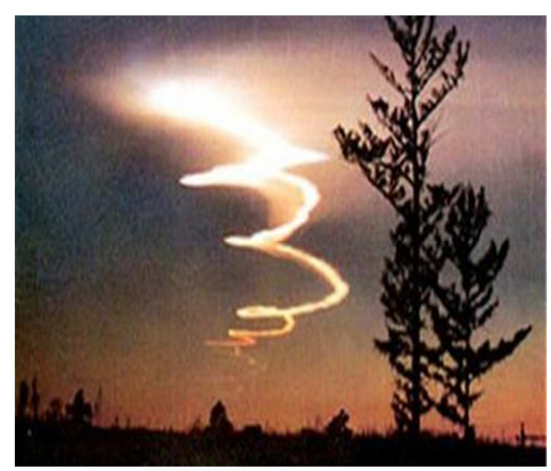

Figure 2. Earth's polar spiral currents.

Since the cloud clusters involved in polar vortex are numerous and revolve downward rapidly in a spiral manner, a series of thick spiral cloud bands can be formed, which facilitate not only the downward flow of heavier negatively charged water droplets but also the transfer of charge. Hence, this kind of cloud band is a good circuit with excellent electrical conductivity. In addition, with a large number of cloud clusters gathering and rapidly plunging in polar vortex, these cloud clusters will become more and more dense and more massive, therefore it is easy to have violent frictions and collisions among clouds clusters, producing frequent electrical discharge or thunderstorms. Each electrical discharge or thunderstorm acts as an electrostatic motor, which can send currents to the upper portion of the vortex and the lower portion of the vortex, forming a series of electrical currents along the spiral cloud bands, as is shown in Figure 2. These spiral currents then produce a synthesized magnetic dipole at Earth's North and South Poles respectively, adding the local non-dipole magnetic fields produced by the atmospheric circuits around the clouds there, finally producing the present geomagnetic field.

In the course of the moon's orbit around the Earth, the Moon has a gravitational pull on both polar vortices, the polar vortices are then deflected, producing geomagnetic declination, which varies with the position of the Moon [3].

Since the polar vortices producing dipole magnetic field are located at the poles of the Earth, the intensity of the geomagnetic field reaches its maximum near North America, Siberia and Antarctica, while it reaches the minimum in the Pacific Ocean near the equator and Central South America. This shows that the spatial distribution of geomagnetic field is not uniform.

In addition, during the Moon's orbiting around the Earth, just as the Moon can cause ocean tides, the Moon has also a gravitational effect on polar vortices. When the Moon approaches a polar vortex, the Moon can tilt or break the polar vortex, even draw some sub cyclones out of the polar vortex, making the dipole magnetic field produced by the polar vortex change. Some of the poured sub cyclones may turn into cyclones with opposite flow direction. which can generate reversed magnetic fields. When the superposition of the generated dipole magnetic fields of opposite direction cancels out the original dipole magnetic field, it makes geomagnetic field reverse.

Additionally, some sub cyclones poured out of the polar vortex, accompanied by the stratospheric flow, move in the direction of the Moon's gravity. When they encounter the high temperature airflow from the ocean surface, they immediately intensify into typhoons or hurricanes. Because these typhoons or hurricanes also produce a certain intensity of magnetic field, with the rapid rotation of the Sun from west to east and the rotation of the Earth from west to east, under the action of the tidal force of the Sun, the magnetic fields of these storms drift westward.

\section{Formation and Characteristics of Mercury's Magnetic Field}

Mercury is one of the eight planets in the solar system, and it is the closest planet to the sun. But its mass is only $3.302 \times$ $10^{23} \mathrm{~kg}$, which is similar to the mass of some satellites, so it can only absorb very thin atmosphere, and its rotation speed is very slow. Hence, it should not have a magnetic field. 
However, the space probe Mariner 10 launched by NASA in the 1970s found that Mercury did exist a weak magnetic field, which is probably a dipole magnetic field like the geomagnetic field, surrounding Mercury, but the magnetic field in the northern hemisphere is much stronger than that in the southern hemisphere. Because Mercury's magnetic field is very similar to the Earth's magnetic field, and its polarity is the same, that is, the south pole of Mercury's magnetic field is in the northern hemisphere of Mercury, and its north pole is in the southern hemisphere. Therefore, some people believe that the origin of Mercury's magnetic field should be similar to that of the Earth's magnetic field, that is, the flow of conductive fluid in the core of the star produces Mercury's magnetic field [4, 5]. However, such a hypothesis cannot explain the magnetic difference between the two hemispheres.

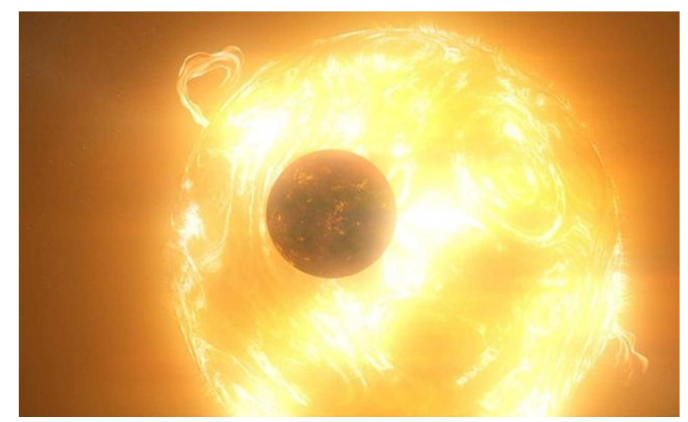

Figure 3. Mercury is magnetized by the Sun's magnetic field.

Since Mercury's internal magnetic field hypothesis cannot be justified, we can only probe the cause of Mercury's magnetic field from its external environment. However, the mass of Mercury is about the same as that of a large satellite, it is difficult for Mercury to accumulate large amounts of gas molecules to form a thick atmosphere, especially Mercury is very hot during the day, the average surface temperature is $179^{\circ} \mathrm{C}$ and the highest is $427^{\circ} \mathrm{C}$, making Mercury lack water molecules in its atmosphere, so Mercury has a very thin atmosphere. In addition, the rotation speed of Mercury is too slow to form atmospheric vortex, therefore, it is impossible to form a dipole magnetic field as the main body of Mercury's magnetic field. Hence, Mercury's magnetic field can only be generated by the interplanetary environment. In fact, Mercury is a planet that goes around the sun, and it's also the closest planet to the sun, whose perihelion is $46001200 \mathrm{~km}$ away from the sun and apogee is $69816900 \mathrm{~km}$ away from the sun. Since Mercury has only an extremely thin atmosphere, under the Sun's irradiation, the surface temperature of Mercury can reach $427^{\circ} \mathrm{C}$. Hence, in the process of Mercury moving around the Sun, a lot of heat energy is accumulated inside mercury. As a result, the internal material of Mercury is fused and differentiated, forming a planet of metals and metal compounds. The exploration data of Mercury also show that Mercury is about $70 \%$ metal and 30\% silicate, so Mercury is a kind of easily magnetized star. Under the effect of the solar wind, the Sun's strong magnetic field can be stretched further than the orbit of Neptune, so Mercury is completely surrounded by the Sun's magnetic field and has been magnetized by the Sun's magnetic field, as shown in Figure 3.

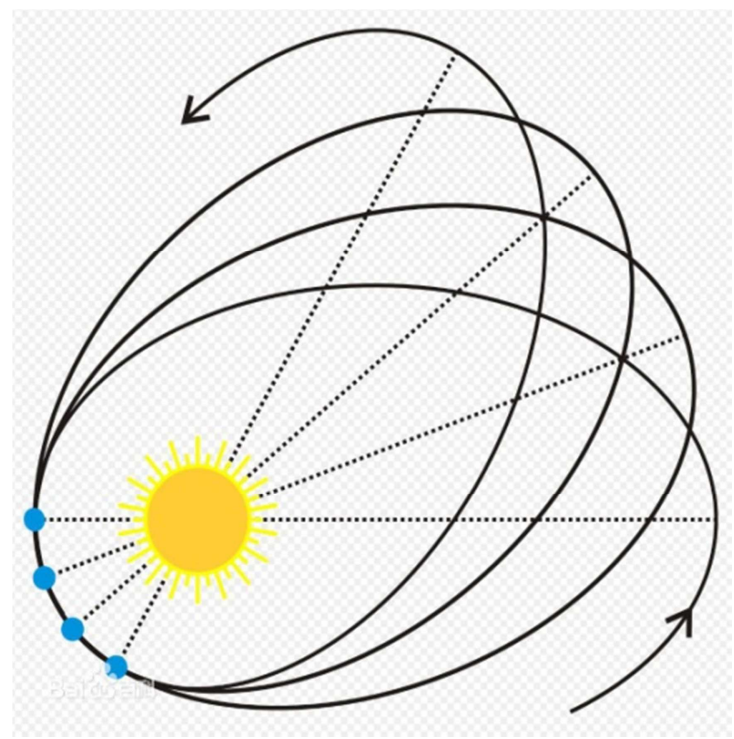

Figure 4. Mercury's orbit around the Sun.

However, Mercury has the largest orbital eccentricity of all planets in the Solar System, its distance from the Sun varies greatly. When it is at perihelion, it is only $46001200 \mathrm{~km}$ away from the Sun, while when it's at apogee, it's $698166900 \mathrm{~km}$ away from the Sun, as is shown in Figure 4. In each cycle of Mercury's orbit around the Sun, it is only near perihelion that it is magnetized by strong solar magnetic field, and when Mercury is near perihelion, the northern hemisphere of Mercury receives more solar electromagnetic radiation than the southern hemisphere, as is shown in Figure 2, this results in a stronger magnetic field in the northern hemisphere than in the southern hemisphere.

\section{Formation and Characteristics of Venus' Magnetic Field}

Venus is one of the eight planets in the Solar System, and it is the second closest planet to the Sun. In addition, it is the closest planet to the Earth, and Venus, like the Earth, is also a terrestrial planet. In addition, its mass and size are similar to that of the Earth, therefore, it should also have a strong magnetic field like the Earth. However, this is not the case, the Earth has a relatively strong magnetic field, while Venus has almost no magnetic field or at most has a very weak magnetic field. Why? This is a question that many scientists, including astronomers, have been searching for $[6,7]$.

The reason why scientists have not been able to answer this question is that they all believe that Venus' magnetic field is generated by the convection in its liquid nuclei, not that the planet's magnetic field is generated by the external atmosphere. Although Venus has a thick atmosphere like the Earth, its rotation period is 243 days, much slower than the Earth's rotation period of 1 day, so its rotation speed is too slow to form polar vortices, which is needed to produce 
dipole magnetic field. In addition, although Venus is also close to the Sun, it is 108.2 million km away from the Sun, more than twice the distance between Mercury's perihelion and the Sun. Hence, the magnetic field of the Sun has little effect on the magnetization of Venus, making the magnetic field of Venus be extremely weak, even 0 .

\section{Formation and Change of Mars' Magnetic Field}

Mars is one of the eight planets in the Solar System, its mass is about 1 / 9 of the mass of the Earth; structurally, it belongs to terrestrial planets as the Earth. The magnetic field of Mars should be maintained at $10 \% \sim 15 \%$ of the Earth's magnetic field, but the intensity of Mars' magnetic field detected by Mars probes is only $0.1 \% \sim 0.2 \%$ of the geomagnetic field, and such weak magnetic fields are only found in the oldest craters [8]. These magnetic fields may be remnants of the early magnetic fields. This strange property of Mars' magnetic field is puzzling. For the formation and evolution of Mars' magnetic field, some scientists believe that Mars is like the Earth, that is, the magnetic field once existed on Mars was also generated by the convection of conductive fluid inside the planet, but there is no evidence for this conjecture. Another explanation has been proposed for the disappearance of Mars' magnetic field: Within a million years, 15 giant impacts occurred coincident with the disappearance of the global magnetic field. The impact heating associated with the largest basins (diameters $>2500 \mathrm{~km}$ ) can cause the global heat flow at the core-mantle boundary to decrease significantly, such a reduction in core heat flow may have led to the cessation of the Martian dynamo [9]. But over time, the temperature of the Martian mantle will fall back, the convection inside the planet will continue, Mars's magnetic field will recover again, how can Mars' magnetic field disappear forever? It can be seen that the theory that asteroids impact on Mars causes the disappearance of Mars magnetic field is also incredible.

The reason why the existing hypotheses about the formation of Martian magnetic field cannot be justified is that they are all endogenous, they haven't probed the origin and evolution of the Martian magnetic field from the external environment of Mars. In fact, in the early days of Martian formation, it was able to absorb a variety of gas molecules, including carbon dioxide and water molecules, to form an atmosphere of a certain thickness. Because Mars is far away from the Sun, its surface temperature is very low, especially the temperature of the two poles is often lower than the melting point of ice $-78^{\circ} \mathrm{C}$. Hence, a large number of carbon dioxide and water molecules are adsorbed on the Martian polar regions, forming ice sheets several kilometers thick, as is shown in Figure 5. Under the influence of such a thick ice sheet, the air in the low air at both poles of Mars is extremely cold. So just as polar vortices can be formed during the rotation of the Earth, polar vortices can also be formed during the rotation of Mars. But during the day, the poles are exposed to the Sun, it is difficult for the air at the poles to condense into water droplets, therefore it is difficult for polar vortices to form strong spiral current, hence no apparent magnetic field or aurora can be detected. Because of the different appearance of Mars at night and during the day, Mars has been known as " bewilder" since ancient times.

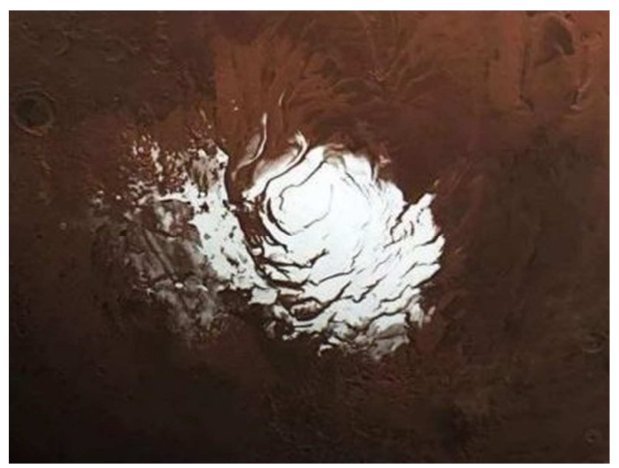

Figure 5. Martian ice sheet.

During the first 500 million to1 billion years of Mars formation, polar vortices existed for a long time. The dipole magnetic field produced by the polar vortices has a long-term magnetization effect on the Mars' surface, therefore a magnetized crust on the surface of Mars has been formed. But during Mars' revolution around the Sun, it accretes dust and gas near its orbit, making Mars' mass increase. With the continuous increase in the mass and volume of the Mars, the heat inside the Mars accumulated continuously, including the heat caused by the air flow friction generated by the high-speed rotation of the Mars around the Sun, the heat generated by the Mars' own gravitational contraction process, the heat generated by the chemical reactions inside the original Mars, etc. It is just the original energy accumulated to a certain extent that made the magma pool form under the surface of Mars, resulting in intense magmatic activities and enough heat, making a large part of Mars Polar ice sheet melt into water. The melting of the Martian polar ice sheet greatly weakened the polar vortex, therefore the magnetic field has been weakened accordingly. Especially in the northern part of Mars, there are large-scale lava activities in the lowlands or volcanic areas, the ice sheet melted a lot there, therefore no polar vortex could be formed, causing the dipolar field disappeared.

In addition, even if there are weak atmospheric vortices on Mars, but as Phobos and Deimos orbit Mars several times a day, their gravitational pull on the cyclones will destroy them easily, so the magnetic field on Mars is almost zero. Only when Mars grows to a large planet like Earth or Jupiter, the atmospheric vortices on it being strong enough to prevent the escaping of gas molecules and not to be easily destroyed by its satellites, can it maintain an enduring magnetic field.

\section{Formation and Change of Jupiter's Magnetic Field}

NASA's Juno spacecraft revealed that Jupiter has an 
internal magnetic field similar to the geomagnetic field [10-12]. This is surprising since numerical simulations have shown that the vastly different interiors of terrestrial and gas planets can strongly affect the internal dynamo process. Yet so far no satisfying explanation has been proposed to eliminate the surprise. Hence, the author analyzed the formation and evolution of Jupiter as well as its internal structure and external environment, and found the formation mechanism and change law of Jupiter's magnetic field: During Jupiter's rapid rotation, a series of strong polar vortices are produced at the poles of Jupiter. These vortices contain a series of strong spiral currents, which can form a series of strong dipole magnetic fields. The superposition of these dipole magnetic fields form the original magnetic field of Jupiter. Since Jupiter has many massive satellites, they are constantly rotating around Jupiter, which has a huge impact on Jupiter's magnetic field. When a massive Jupiter satellite approaches a polar vortex, it can tilt, stretch, shear or break the polar vortex, even draw some sub cyclones out of the polar vortex, and some sub cyclones may turn into cyclones with opposite flow direction. Hence, the destruction of Jupiter's satellites will not only weaken the dipole magnetic field produced by the original cyclone, but also generate some reversed magnetic fields, which can counteract part of the original magnetic field. When this kind of Jupiter moons revolve enough times, the superposition of the generated magnetic fields of opposite direction will cancel out the original magnetic field, finally, making Jupiter's magnetic field reverse. Therefore, the north pole of Jupiter's magnetic field is near the geographical North Pole, and the south pole of Jupiter's magnetic field is near the geographical South Pole. Hence, the direction of Jupiter's magnetic field is opposite to that of Earth's magnetic field [13-16].

\section{Conclusions}

The existing theories about the origin of planetary magnetic fields are all based on the internal generator theory of geomagnetic field, which is still in doubt, therefore they are led astray, unable to explain the formation and change of the planetary magnetic fields in the solar system. Thus, the author analyzed the formation and evolution of each planet in the solar system as well as its internal structure and external environment to find the following important law about the formation and change of planetary magnetic fields: if a planet similar to Mercury or Venus is close to its parent star and rotates very slowly, it cannot produce polar vortex and therefore dipole magnetic fields; if a planet similar to Earth, Mars or Jupiter is far away from its parent star and rotates very quickly, it can produce polar vortex and therefore dipole magnetic fields.

\section{References}

[1] Schubert G, Soderlund K M. Planetary magnetic fields: Observations and models [J]. Physics of the Earth \& Planetary Interiors, 2011, 187 (3-4): 92-108.

[2] Jones, Chris A. Planetary Magnetic Fields and Fluid Dynamos [J]. Annual Review of Fluid Mechanics, 2011, 43 (1): 583-614.

[3] Cuixiang Zhong. Movement of Polar Vortices and its Close Relationship with Global Climate Change [J]. American Journal of Earth and Environmental Sciences, 2019-07-04.

[4] Uno, H. (2009). New constraints on Mercury's internal magnetic field (T). University of British Columbia. Retrieved from https://open.library.ubc.ca/collections/ubctheses/24/items/1.00534 68 .

[5] GOLDSTEIN, M., NESS, N. \& WASILEWSKI, P. Mercury's magnetic field. Nature 262, $741 \quad$ (1976). https://doi.org/10.1038/262741a0.

[6] Luhmann J G, Russell C T. Venus: Magnetic field and magnetosphere [M]. Springer Netherlands, 1997.

[7] Nimmo F. Why does Venus lack a magnetic field? [J]. Geology, 2002, 30 (11): 987-990.

[8] Russell C T, Luhmann J G, Spreiter J R, et al. The magnetic field of Mars - Implications from gas dynamic modeling [J]. Journal of Geophysical Research Space Physics, 1984, 89 (A5): 2997-3003.

[9] Roberts, J. H. Lillis, R. J. Manga, M. Giant impacts on early Mars and the cessation of the Martian dynamo [J]. Journal of Geophysical Research Planets, 2009/04/23.

[10] Zaghoo Mohamed, Collins G. W. Size and Strength of Self-excited Dynamos in Jupiter-like Extrasolar Planets [J]. The Astrophysical Journal, 862 (1), March 2018.

[11] Yue-Kin Tsang; Chris A. Jones. Characterising Jupiter's dynamo radius using its magnetic energy spectrum [J]. Earth and Planetary Science Letters, 2020-01-15.

[12] Jones Chris. Jupiter's magnetic field revealed by the Juno spacecraft [J]. Nature, 2018.

[13] K. M. Moore, H. Cao, J. Bloxham, D. J. Stevenson, J. E. P. Connerney \& S. J. Bolton. Time variation of Jupiter's internal magnetic field consistent with zonal wind advection [J]. Nature Astronomy, vol. 3, pp. 730-735 (2019).

[14] J. Wicht; T. Gastine; L. D. V. Duarte; W. Dietrich. Dynamo action of the zonal winds in Jupiter [J]. Astronomy \& Astrophysics, 2019-09-19.

[15] Christopher Crockett. Jupiter's magnetic field is surprisingly weird [J]. Science News, 2018-09-29.

[16] Cuixiang Zhong. Formation and Change of Jupiter's Magnetic Field [J]. American Journal of Astronomy and Astrophsics. Vol. 8, No. 2, 2020, pp. 35-38. doi: 10.11648/j.ajaa.20200802.14. 